\title{
OPTICAL COHERENCE MICROSCOPY FROM TISSUE TO CELL
}

\author{
Theo Lasser ${ }^{1}$, Martin Villiger ${ }^{1}$, Christophe Pache ${ }^{1}$, Arno Bouwens ${ }^{1}$, Corinne Berclaz ${ }^{1}$, \\ Daniel Szlag', Marcel Leutenegger ${ }^{1}$, Tristan Bolmont ${ }^{1}$ \\ ${ }^{1}$ Laboratoire d'Optique Biomédicale, Ecole Polytechnique Fédérale de Lausanne, \\ CH-1015 Lausanne, Switzerland \\ Email: theo.lasser@epfl.ch
}

KEY WORDS: Optical Coherence Microscopy, diabetes, Alzheimer, cell imaging

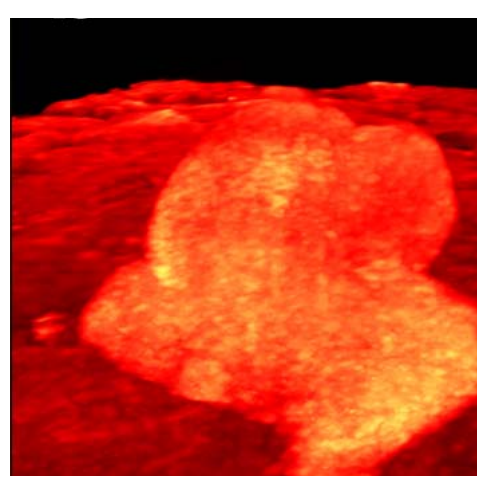

Fig.1 Islets of Langerhans

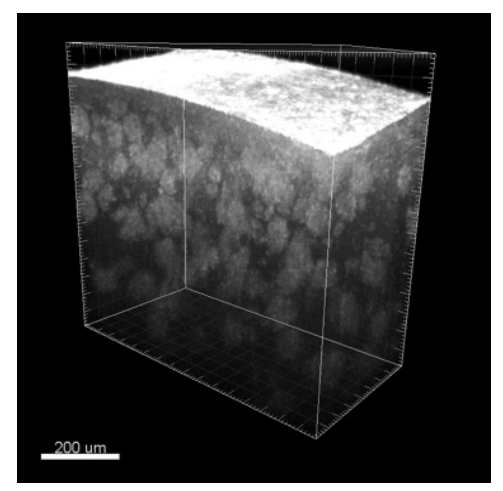

Fig. 2 Amyloid plaques

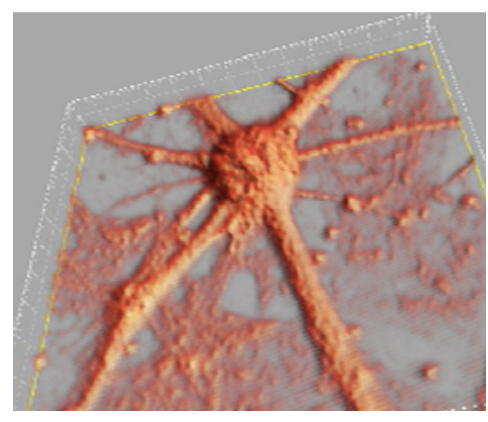

Fig. 3 Neuron

\section{Introduction}

Extended-focus Optical Coherence Microscopy (xf-OCM) offers new roads for in-vitro as well in-vivo imaging in life sciences and medicine. We show the latest achievements based on this imaging modality with new applications for diabetes and Alzheimer's disease research.

\section{Results and outlook}

In diabetes research a quantitative label-free imaging of islets of Langerhans is of high importance (see Fig. 1). xf-OCM circumvents the compromise between lateral resolution and depth of field by us of a Bessel-like illumination beam. The high sensitivity and parallel depth profiling of Fourier domain optical coherence tomography are preserved, and combined with a high isotropic resolution of $1.5-2 \mu \mathrm{m}$. A semi-automatic islet detection algorithm allows to measure in vitro and vivo islet size, position, contrast and homogeneity. Finally, xfOCM was used to assess the murine pancreas in situ and in vivo, visualizing pancreatic lobules, ducts, blood vessels and individual islets of Langerhans.

As a next field of application, we present xf-OCM as an instrument for label-free investigation of the pathology of Alzheimer's disease (AD) in a live mouse model. Both structural imaging of cerebral amyloid- $\beta$ plaques (see Fig. 2 ), a hallmark of $\mathrm{AD}$, and high-resolution functional imaging of the brain's vascular network are demonstrated. Finally, a strategy to perform label-free longitudinal studies on the mouse brain using $\mathrm{xfOCM}$ is shown.

Recently we upgraded the xf-OCM principle to darkfield Optical Coherence Microscopy (df-OCM) to provide further contrast enhancement applied to cell imaging (see Fig. 3). The darkfield effect suppresses direct reflection to a large extent, while keeping the benefits of fast imaging speed and high sensitivity of detection in the Fourier domain.

Finally, we show that tissue, cell and sub-cellular structures can all be visualized based on these coherent imaging methods and therefore provide a variety of information with high spatial and temporal resolution. Across these different examples, we intend to provide new applications and innovative imaging technologies. 